\title{
Rotation of B[e] Supergiants, Luminous Blue Variables and Wolf-Rayet Stars
}

\author{
Philippe Eenens \\ Departmento de Astronomía, Universidad de Guanajuato, Apartado \\ 144, Guanajuato, Gto 36000, Mexico E Institut d'Astrophysique, \\ Université de Liège, Belgium
}

\begin{abstract}
A brief review of the observational properties of evolved massive stars is presented. In the pursuit of clues for their rotation, formidable difficulties are encountered. At the same time, common features emerge between $\mathrm{B}[\mathrm{e}]$ supergiants, Luminous Blue Variables and Wolf-Rayet stars. Several indications of rapid rotation are found, mostly indirect ones, in each class. It is shown that fast rotation can probably survive the mass loss events which characterize the late evolution of massive stars.
\end{abstract}

\section{Introduction}

Among hot massive stars, three classes are notorious for their strong, dense stellar winds: $\mathrm{B}[\mathrm{e}]$ supergiants, Luminous Blue Variables (LBVs) and WolfRayet (WR) stars. These stars share several peculiar spectral properties, some stars having been observed to cross the boundaries between classes. For these and other reasons, we argue that they are in closely related evolutionary stages (Morris et al. 1996). Could the differences between Be, B $[\mathrm{e}]$ and LBVs classes be explained by rotation, at least to some extent? The faster a star rotates, the more its wind will loose its spherical symmetry, creating an equatorial density enhancement (a 'disk'). Lenorzer, de Koter \& Waters (2002) examined the ratios of near-infrared lines to describe the circumstellar gas in massive stars. They show that Be stars, B[e] supergiants and LBVs form well-separated groups (Fig. 1). In their diagram, the Be stars cluster in a region of optically thick emission, typical of disks. At the other extreme, LBVs are close to the region where optically thin emission dominates. $\mathrm{B}[\mathrm{e}]$ supergiants occupy an intermediate region.

The powerful winds from massive stars have a high impact on the physics and the evolution of these stars (see e.g. Lamers and Cassinelli 1999; Chiosi and Maeder 1986). In turn, stellar rotation can drastically affect stellar winds (Lamers 2004). Thus rotation can have a strong influence on stellar evolution, not only through effects such as chemical mixing and transport of angular momentum (Maeder 2004), but also indirectly, because it modifies the stellar wind, which is itself an important factor in the evolution of a massive star.

It is therefore highly desirable to determine the rotation rate of these stars. This is even more so because of the need to support the hypothesis that Gamma Ray Bursts originate from rapidly rotating WR stars (Woosley \& Heger 2004). 


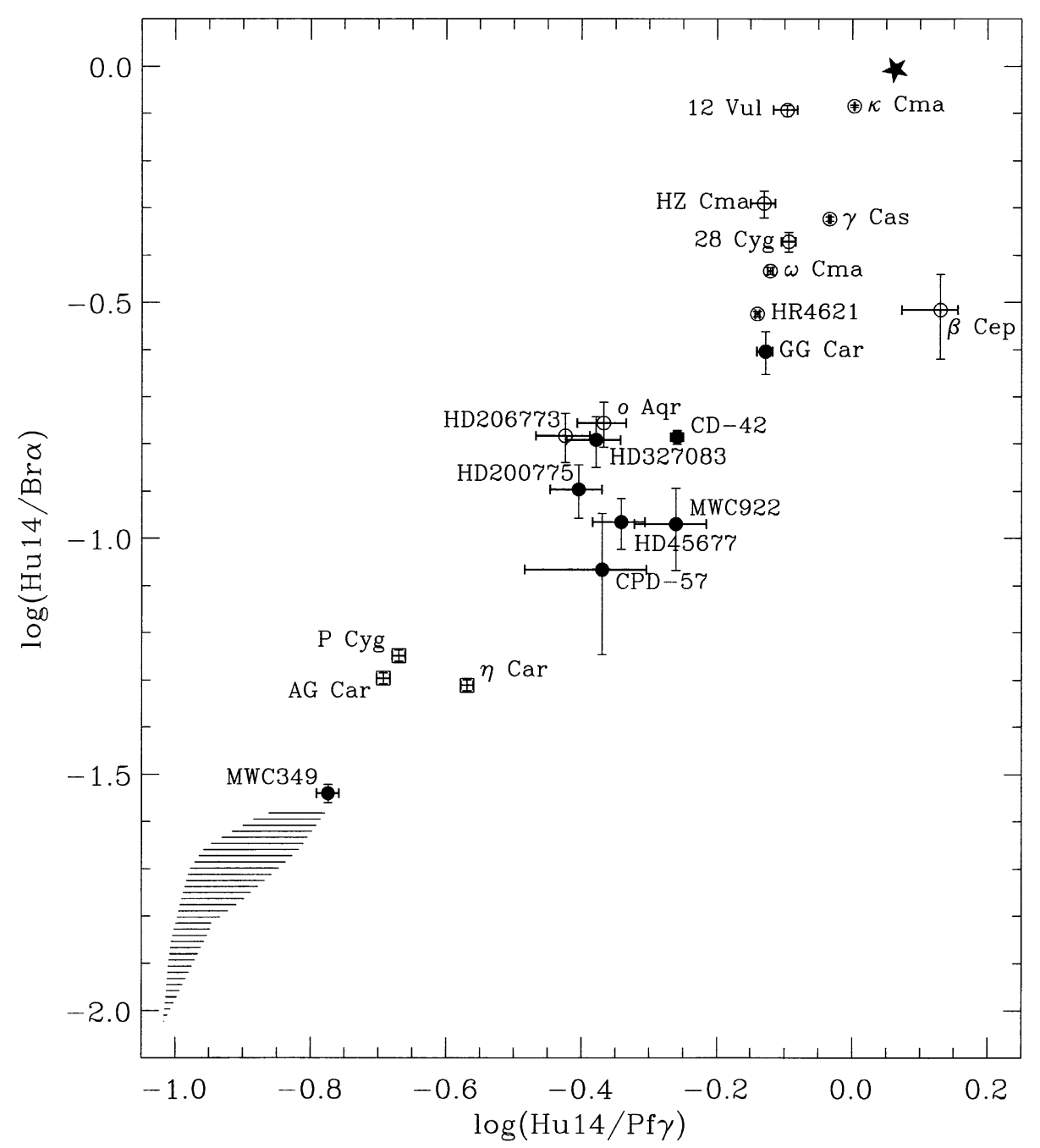

Figure 1. Hydrogen infrared recombination line ratio diagram, $\mathrm{Hu}(14-$ 6)/Br $\alpha$ versus $\mathrm{Hu}(14-6) / \mathrm{Pf} \gamma$, showing the location of LBVs (squares), B[e] (filled circles) and Be stars (open circles) with respect to optically thin (stripes) and thick emission (thick asterisk) (from Lenorzer et al. 2002). 
Unfortunately, the very existence of dense winds around massive stars means that their photosphere is hidden from our view. As a consequence the direct measurement of $v \sin i$ becomes an almost impossible task. Here we will recount recent efforts made to estimate rotation rates of evolved massive stars with strong winds, mainly through indirect methods. Evidence for and implications of rotation of massive stars were presented by Schulte-Ladbeck (1997). An in-depth discussion of observational evidence for massive star rotation in the context of stellar evolution can be found in the review by Maeder \& Meynet (2000).

\section{2. $\mathbf{B}[\mathbf{e}]$ Supergiants}

Reviews on $\mathrm{B}[\mathrm{e}]$ stars can be found in the proceedings of the Paris meeting $(\mathrm{Hu}-$ bert \& Jaschek 1998), the pivotal paper by Lamers et al. (1998) and Zickgraf's habilitation thesis (1998). As first described by Allen \& Swings (1972), the spectra of some early-type stars show both strong Balmer lines in emission and forbidden emission lines of [Fe II] and [O I]. These unusual spectral characteristics are normally associated with the observation of a strong infrared excess and are referred to as the $\mathrm{B}[\mathrm{e}]$ phenomenon. These spectral features have been observed in stars belonging to very different evolutionary groups, such as premain-sequence Herbig Ae Be stars, symbiotic stars and planetary nebulae. Here we will consider only $\mathrm{B}[\mathrm{e}]$ supergiants, which are the most luminous of the stars displaying the $\mathrm{B}[\mathrm{e}]$ phenomenum. To define them, Lamers et al. (1998) propose a luminosity threshold of $\log \mathrm{L}_{\star} / \mathrm{L}_{\odot}>$ 4.0. These stars are also characterized by $\mathrm{P}$ Cygni spectral profiles (indicative of mass loss) and small photometric variations.

Zickgraf (1985) has shown that their spectral lines can be grouped in two very different categories and can be explained in terms of a hybrid atmosphere model (Fig. 2). The narrow, low-excitation emission lines (broadened by a few $10 \mathrm{~km} \mathrm{~s}^{-1}$ ) are believed to form in a cool, dense, slowly expanding equatorial disk wind. The broad, high-excitation absorption lines (with velocities of 1000-2000 $\mathrm{km} \mathrm{s}^{-1}$ ) originate in a hot, fast radiation-driven wind blowing from the stellar poles. This polar wind is stronger than in classical Be stars due to the higher stellar luminosity. Such a structure involving an equatorial disk and a fast polar wind is the clear signature of rotation.

As shown by Allen \& Swings (1976), the observation of an infrared excess due to hot $(\sim 1000 \mathrm{~K})$ circumstellar dust is common among $\mathrm{B}[\mathrm{e}]$ supergiants. The radiation field of a $\mathrm{B}[\mathrm{e}]$ supergiants is an unlikely environment for dust. However this difficulty is overcome if $\mathrm{B}[\mathrm{e}]$ supergiants are rapidly rotating, as depicted in the hybrid model. In this case, dust formation is made possible through the coincidence of several factors: the shielding of the ultraviolet radiation by the circumstellar disk, its high density and its low temperature.

In the infrared spectrum of $\mathrm{B}[\mathrm{e}]$ supergiants the $\mathrm{CO}$ first overtone band, typical of circumstellar disks, has also been observed (McGregor, Hillier \& $\mathrm{Hy}$ land 1988; McGregor, Hyland \& McGinn 1989). In S 18, no CO emission was detected by McGregor et al. (1989) but it was present in our 1995 spectrum (Morris et al. 1996), which suggests that the equatorial outflow or the physical conditions in the disk are variable. 


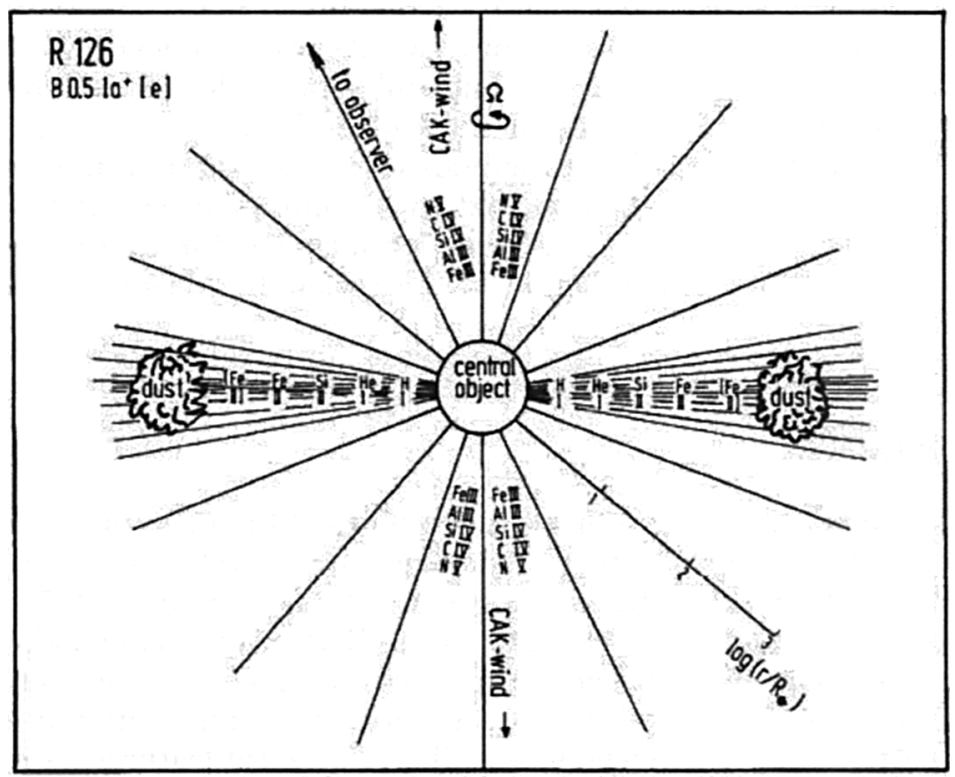

Figure 2. Sketch of the two-component wind model, based on observations of the B[e] supergiant R126 in the LMC (from Zickgraf 1998)

Another indicator, albeit indirect, of fast rotation is the detection of significant intrinsic polarization detected in several $\mathrm{B}[\mathrm{e}]$ supergiants in the Magellanic Clouds (Magalhaes 1992, Schulte-Ladbeck et al. 1994). From these observations, it appears that the circumstellar environment is not spherical and that the objects seen edge-on show higher polarization. Polarization has also been found in Galactic B[e] stars, but their classification as supergiants is more difficult to establish (Zickgraf \& Schulte-Ladbeck 1989). Recent examples are MWC 349A which may well be a pre-main-sequence object (Meyer, Nordsieck \& Hoffman 2002); MWC 623, a B[e]/K binary, believed to be slightly evolved beyond the main sequence (Zickgraf 2001); and HD 87643 for which there are some indications that it is an evolved, luminous object (Oudmaijer et al. 1998). In view of these converging arguments, and to seek support for the hybrid model, Zickgraf et al. (1986) have set to the task of determining the $v \sin i$ of photospheric lines, despite the difficulty to find suitable lines. Synthetic spectra were constructed with appropriate effective temperature, effective gravity and microturbulence. For the B[e] supergiant Hen S93, the best fit to the Mg II $\lambda 4481$ line yields $v \sin i$ $=65 \mathrm{~km} \mathrm{~s}^{-1}$. A $v \sin i$ of $150 \mathrm{~km} \mathrm{~s}^{-1}$ is obtained from the fitting of the He I $\lambda 4009$ line in the spectrum of the $\mathrm{B}[\mathrm{e}]$ supergiant $\mathrm{R} 50$, which is believed to be seen nearly edge on. The He I $\lambda 5875$ line in the B[e] supergiant R 66 , a star probably observed almost pole-on, gives a $v \sin i$ of $50 \mathrm{~km} \mathrm{~s}^{-1}$. These authors have also obtained estimates of the rotation velocity from the determination of the effective gravity in the equatorial zone. They derive rotational velocities of 
240, 224 and $204 \mathrm{~km} \mathrm{~s}^{-1}$ for Hen S22, R 82 and R 50 respectively. These values are consistent with the projected velocities obtained with the other method.

It is therefore reasonable to conclude that most, and perhaps all, $\mathrm{B}[\mathrm{e}]$ supergiants are rapidly rotating stars, with velocities above $100 \mathrm{~km} \mathrm{~s}^{-1}$.

\section{Luminous Blue Variables}

LBVs were the object of a Paris workshop (Nota \& Lamers 1997) and various reviews (e.g. Humphreys \& Davidson 1994; Nota et al. 1995). LBVs are very massive (a few $10 \mathrm{M}_{\odot}$ ), very luminous $\left(\mathrm{L}_{\star}>10^{6} \mathrm{~L}_{\odot}\right)$ stars. They are believed to be evolved stars, in a short transition stage between Main-Sequence $\mathrm{O}$ stars and WR stars. Their evolutionary relation to $\mathrm{B}[\mathrm{e}]$ supergiants is still unclear.

LBVs undergo violent episodes of mass loss, with time scales of decades (and a magnitude increase of 1 or 2$)$ or longer $(\Delta \mathrm{m}>2: \eta$ Car, $\mathrm{P}$ Cyg). They also show variability on shorter time scales. These stars can remain for long periods of time in either of two different phases. During the quiescent phase, they are visually fainter, with a spectrum of a hot supergiant or of an Of/WN9 star. During outburst, they become visually brighter, while they exhibit the spectrum of a cooler A-F supergiant.

Can LBVs keep high rotation rates after presumably loosing much angular momentum during episodes of mass loss? There are no measurements of $v \sin i$ but indirect evidence for fast rotation has been investigated in several LBVs. This evidence will be briefly reviewed here for some prominent LBVs, leaving aside axisymmetric nebulae, which are covered elsewhere in these proceedings (Nota 2004).

The LBV star AG Car displays very large variations of its linear polarization (Schulte-Ladbeck et al. 1994). The polarization is found to vary along a preferred position angle that is co-aligned with the major axis of the circumstellar nebula. This is interpreted as a symmetry axis of an anisotropic stellar wind. Diverse features in its spectrum are attributed to either component of a two-component wind (Leitherer et al. 1994): the optical emission lines come from a dense wind, probably located near the equator, while ultraviolet P-Cygni type profiles indicate the presence of a less dense wind, believed to originate from the stellar poles. On the other hand, $\mathrm{CO}$ overtone bands observed in its infrared spectrum reveal the presence of circumstellar dust (Nota et al. 2002). We have shown in the case of another LBV, HR Car, that these bands are detected during quiescent phases only and we have argued that the dust must be located in a disk, because a uniform spherical distribution would not meet the density requirements. A disk would aslo provide the necessary shielding from the harsh stellar radiation (Morris et al. 1997). This picture is consistent with the predictions of the bistable wind model (Lamers \& Pauldrach 1991; Lamers 2004). It is similar to that put forward for $\mathrm{B}[\mathrm{e}]$ supergiants. To support the close relation between both stellar types we note that our CTIO infrared spectra (2 $2.4 \mu \mathrm{m}$ ) of the LBVs HR Car and AG Car are very similar to those of the B[e] supergiants GG Car and HD 72754 (Morris et al. 1996).

Spectropolarimetric and spectroscopic studies have revealed that other LBVs have non-spherical winds. This is the case for HR Car (Clampin et al. 1995; Parthasarathy, Jain \& Bhatt 2000) and R 127 (Schulte-Ladbeck et al. 1993). In 
the case of P Cyg, Nordsieck et al. (2001) propose that the star is seen under an inclination angle lower than $18^{\circ}$. Clumps would be ejected near the equatorial plane (almost aligned with the plane of the sky), in agreement with the observations of a very clumpy wind and large polarimetric variations. Smith \& Davidson (2001) found shocked gas in the polar lobes of $\eta$ Car and interpreted it as an indication of the presence of a fast polar wind. HD 326823 is another interesting star, as it is believed to be in transition between the LBVs and WR phases (Borges Fernandes et al. 2001). Its He I spectrum displays double-peak profiles reminiscent of a disk structure.

\section{Wolf-Rayet Stars}

Wolf-Rayet stars are evolved massive stars, exhibiting in their spectra products of hydrogen and helium burning $(\mathrm{He}, \mathrm{N}, \mathrm{C}, \mathrm{O})$. In addition to helium lines, WN spectra are dominated by nitrogen emission lines, and WC spectra by carbon lines. The very broad emission lines originate from fast, dense winds with velocities of several thousand $\mathrm{km} \mathrm{s}^{-1}$. Many WR stars are found in WR+O binaries, where the collision between the winds of the two massive stars create strong shocks. It has been suggested that evolutionary paths from $\mathrm{O}$ stars to $\mathrm{WR}$ stars depend on the original mass: the most massive $\mathrm{O}$ stars evolve directly into the hydrogen-rich WN6-WN7 WR subtypes; less massive O stars go through an LBV stage before ending as WN8 types; the least massive ones become red supergiants before producing either of the remaining WR subtypes. (For recent reviews on WR stars, see the proceedings of IAU Symposium 212 edited by van der Hucht, Herrero \& Esteban 2003).

The latest Gamma Ray Bursts models require the existence of fast-rotating WR stars (Woosley \& Heger 2004). However observational data have provided very little information on the rotation rate of observed WR stars. Most WR spectra entirely lack photospheric absorption lines, as the photosphere is hidden by their dense winds and the spectrum is dominated by broad emission lines. In the past, there have been several attempts to derive rotational velocities in the few cases where absorption lines were visible. Indeed, HD 193077 (WR 138) was believed to be a single WR star with a $v \sin i$ of $500 \mathrm{~km} \mathrm{~s}^{-1}$ (Massey 1980). In the binary system HD 92740 (WR 22) the absorption lines are moving in phase with the WR companion (Niemela 1973). This is also the case in HD 9974 (WR 3) where the line widths yielded a $v \sin i$ between 150 and $200 \mathrm{~km} \mathrm{~s}^{-1}$ (Massey \& Conti 1981; Moffat et al. 1986). However WR 138 has since been shown to be a binary system and its broad absorption lines have been attributed to the $\mathrm{O}$ star companion (Annuk 1991). In the two remaining cases the absorption lines have been shown to originate not in the photosphere but in the wind (Rauw et al. 1996; Marchenko et al. 2004).

In the absence of direct measurements, indirect evidence for rotation has been sought through studies of nebulae (Nota 2004), polarimetry, radio observations and rapid line-profile variations.

A reduction of the polarization at emission-line wavelengths has been detected in 20 percent of the WR stars observed by Harries, Hillier \& Howarth (1998). This 'line effect' is attributed to asymmetric flattened winds. Inclination angles alone do not explain the low number of line-effect stars, i.e. not 


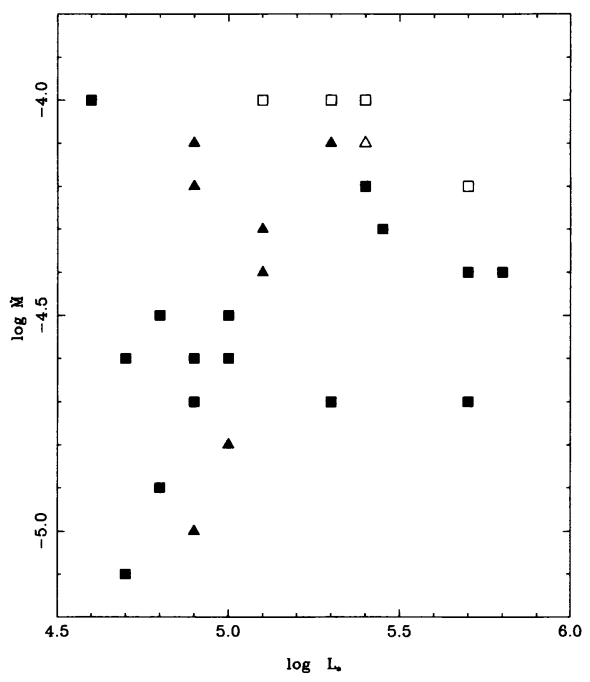

Figure 3. Mass-loss rates of WN (squares) and WC stars (triangles) versus luminosity. The non-spherical winds (open symbols) have systematically higher luminosities than spherical winds (filled symbols) (from Harries et al. 1998)

all WR stars rotate rapidly. The line-effect stars have the highest mass-loss rates among WR stars of a given luminosity (Fig. 3). Also, their winds tend to have the largest velocities for a given subtype. This is consistent with the rotational origin of the line effect, as the rotation will increase both mass-loss rates and wind velocity. The rapid variability observed in emission lines of EZ Canis Majoris (WR 6), of the order of a few days, had led to the controversial suggestion that it is a WR binary with a collapsed companion. In order to test the rotation or binary nature of WR 6, Harries et al. (1999) have obtained intensive polarimetry of this star. The continuum polarization does not show the sinusoidal-shaped curve expected for a close binary. On the contrary, the magnitude of the continuum polarization is consistent with an asymmetric structure of the wind. They derive an equator/pole density ratio of 3 . The variability in the line polarization favors the corotating model, i.e. the rotational modulation of a small number of large-scale structures. These findings suggest that WR 6 is indeed a single, rapidly-rotating star.

Recent radio observations are confirming this view. The ovoidal bubble observed around WR 6 are probably caused by an anisotropic wind. Although the VLA observations do not clearly resolve the wind, they place an upper limit to its anisotropy: the ratio between the major axis and the minor axis does not exceed 2 (Contreras et al. 2000). A collapsed companion would produce a non-thermal, variable wind. However, these authors do not observe variations and find that the wind of WR 6 is thermal at $9000 \mathrm{~K}$, with a spectral index between 0.6 and 0.8 . This rules out a collapsed companion. 
Intense optical spectroscopic monitoring has also contributed to a better understanding of the line-effect stars. They all display strong line-profile variability. We have shown that the variability of HD 191765 (WR 134) is epoch-dependent but there is an underlying period (2.3 day) which remains coherent over time scales of years (Morel et al. 1999a). This coherence requires a steadfast underlying mechanism, such as rotation or binarity but we have shown that the X-ray fluxes are not compatible with the presence of a collapsed companion (Morel et al. 1998; 1999a). The same is true for WR 6 which has a period of 3.77 day (Morel et al. 1998; Georgiev et al. 1999) although it has been suggested that it could have a M-type star companion (Skinner et al. 2002).

In the infrared spectrum of WR 134, we have detected a strong subpeak on top of the He I line at $1.083 \mu \mathrm{m}$ (Eenens 1992). The subpeak is seen at a constant Doppler position in each observation. Although the sparse data cannot rule out a periodic wavelength shift of the subpeak, its constancy would certainly be consistent with a polar jet, in agreement with the model proposed by Vreux, et al. (1992).

The mysterious WR 1 (HD 4004) has also been the target of recent monitoring campaigns, which revealed some unexpected characteristics. The line profile variations do not follow the general wind law and the projected velocities do not scale with line width (Niedzielski 2000). This suggests a two-component wind, related to a significant rotation. In this model, the irregular brightenings (observed by Morel et al. 1999b) are explained by the sudden formation of non-stable disk structures. These irregularities propagate outwards in the wind at a lower speed than the bulk of the wind. The observed random X-ray variability results from the interaction of the two winds. As WR 1 displays many similarities with WR 6 and WR 134, an extended spectroscopic monitoring is of importance to continue the search for a period in the line profile variability (LPV). Polarimetric data are also needed to detect a possible disk flattening.

Can we estimate the rotation rate of WR stars? For WR 6 and WR 134, we can combine the LPV periods, the radii (resp. 1.8 and $4.2 \mathrm{R}_{\odot}$ ) and the mass (resp. 16 and $33 \mathrm{M}_{\odot}$, Hamann \& Koersterke 1998) to infer rotational velocities of 24 and $92 \mathrm{~km} \mathrm{~s}^{-1}$ which correspond to respectively 7 percent and 2 percent of the critical velocity, within the range predicted by theoretical models (Meynet \& Maeder 2003).

Our study of WR 3 (WN3) indicates that rotation, not binarity, is at the origin of its unexpected high hydrogen contents (Marchenko et al. 2004). This is based on the lack of short-period photometric variations, on magnitude constraints for the companion, on the triangular shape of its emission lines, and on the constant blue-shift of its absorption lines.

\section{Conclusions}

The study of B[e], LBV and WR stars reveals common features. Their progenitors, the massive main-sequence stars of type $\mathrm{O}$ and $\mathrm{B}$, are known to rotate very fast. As they evolve, they go through episodes of mass loss, either in gigantic outbursts (in the LBV stage), or extended over long periods of time (WR stage). It seems however that the mass loss phenomenon does not completely take away 
the original angular momentum. We find signs of fast rotation all the way from the main sequence to the WR phases, including the WC subtypes.

Rotation seriously affects the stellar winds. It increases the wind velocity and the mass-loss rate, and modifies the spherical symmetry. A two-component wind is sometimes observed in each class, $\mathrm{B}[\mathrm{e}], \mathrm{LBV}, \mathrm{WR}$. In such cases, the geometry of the mass loss must obviously be more complex than that modelled by spherical geometry, with consequences on the inferred mass-loss rates and on evolutionary models. Estimating the rotation rates will however remain a challenge.

\section{References}

Allen, D.A., Swings, J.-P. 1972, ApJL 10, 83

Allen, D.A. \& Swings, J.P. 1976 A\&A 47, 293

Annuk, K. 1991 in Wolf-Rayet Stars and Interrelations with Other Massive Stars in Galaxies, ed. K.A. van der Hucht \& B. Hidayat, IAU Symp. 143 (Dordrecht, Kluwer) p. 245

Borges Fernandes, M., de Araújo, F.X., Pereira, C.B., Landaberry, S.J.C. 2001 ApJS 136,747

Chiosi, C. \& Maeder, A. 1986, An. Rev. Astr. Ap. 24, 329

Clampin, M., Schulte-Ladbeck, R.E, Nota, A., Robberto, M., Paresce, F. \& Clayton, G.C. 1995 AJ 110, 251

Contreras, M.E., Rodríguez, L.F. \& Arnal, E.M. 2000 RevMexAA 36, 135

Eenens, P.R.J. 1992 in Variable and non-isotropic outflows from stars, ed. L. Drissen, C. Leitherer, \& A. Nota, A.S.P. Conf. Ser. 22 (Provo, Brigham Young University) p. 145

Georgiev, L.N., Koenigsberger, G., Ivanov, M.M. \& cardona, O. 1999 A\&A 347, 583

Hamann, W.-R. \& Koersterke, L. 1998 A\&A 333, 251

Harries, T. J., Howarth, I. D., Schulte-Ladbeck, R. E. \& Hillier, D. J 1999 MNRAS 302, 499

Harries, T.J., Hillier, D.J. \& Howarth, I.D. 1998 MNRAS 296, 1072

Hubert, A.M., Jaschek, C. 1998, B[e] stars (Dordrecht, Kluwer)

Humphreys, R.M. \& Davidson, K. 1994 PASP 106, 1025

Karel van der Hucht, A. Herrero \& C. Esteban 2003, A massive star odyssey: from main sequence to supernova, proc. IAU-Symp. 212 (San Francisco, ASP)

Lamers, H.J.G.L.M. 2004, these proceedings

Lamers, H.J.G.L.M. \& Cassinelli, J.P. 1999, Introduction to stellar winds (Cambridge, CUP)

Lamers, H.J.G.L.M., Pauldrach, A.W.A. 1991 A\&A 244, L5

Lamers, H.J.G.L.M., Zickgraf, F.-J., de Winter, D., Houziaux, L., Zorec, J. 1998, A\&A 340,117

Leitherer, C., Allen, R., Altner, B., Damineli, A., Drissen, L., Idiart, T., Lupie, O., Nota, A., Robert, C., Schmutz, W. \& Shore, S. N. 1994 ApJ 428, 292

Lenorzer, A., de Koter, A. \& Waters, L. 2002 A\&A 386, L5

Meynet, G.; Maeder, A. 2003 A\&A 404, 975

Maeder, A. 2004, these proceedings

Maeder, A., Meynet, G. 2000, An. Rev. Astr. Ap. 2000, 38, 143 
Magalhaes, A.M. 1992 ApJ 398, 286

Marchenko, S.A., Moffat, A.F.J., Crowther, P.A., Chené, A.-N., De Serres, M., Eenens, P.R.J., Hill, G.M., Moran, J., Morel, T. 2004 in prep.

Massey, P. 1980 ApJ 236, 526

Massey, P. \& Conti, P.S. 1981 ApJ 244, 173

McGregor, P.J., Hillier, D.J. \& Hyland, A.R. 1988 ApJ 334, 639

McGregor, P.J., Hyland, A.R. \& McGinn, M.T. 1989 ApJ 223, 237

Meyer, J.M., Nordsieck, K.H. \& Hoffman, J.L. 2002 AJ 123, 1639

Moffat, A.F.J., Lamontagne, R., Shara, M. \& McAlister, H.A. 1986 AJ 91, 1392

Morel, T., St-Louis, N., Moffat, A.F.J., Cardona, O., Koenigsberger, G. \& Hill, G.M. 1998 ApJ 498, 413

Morel, T., Marchenko, S.V., Eenens, P.R.J., Moffat, A.F.J., Koenigsberger, G., Antokhin, I.I., Eversberg, T., Tovmassian, G.H., Hill, G.M., Cardona, O. \& StLouis, N. 1999a ApJ 518, 428

Morel, T., Georgiev, L.N., Grosdidier, Y., St-Louis, N., Eversberg, T. \& Hill, G.M. 1999b A\&A 349, 457

Morris, P.W., Eenens, P.R.J., Hanson, M.H., Conti, P.S. \& Blum, R.D. 1996 ApJ 470, 597

Morris, P.W., Voors, R.H.M., Lamers, H.J.G.L.M. \& Eenens, P.R.J. 1997 in ASP Conf. Ser. 120, Luminous Blue Variables, ed. A. Nota \& H.J.G.L.M. Lamers, p. 20

Niedzielski, A. 2000 A\&A 362, 289

Niemela, V.S. 1973 PASP 85, 220

Nordsieck, K.H., Wisniewski, J., Babler, B.L. et al. 2001 in P Cygni 2000 - 400 years of Progress, ASP Conf. Ser. Vol. 233, ed. M. de Groot (San Francisco: ASP), p. 261

Nota, A. 2004, these proceedings

Nota, A. \& Lamers, H.J.G.L.M. 1997 ASP Conf. Ser. 120 (San Francisco, ASP)

Nota, A., Livio, M. Clampin, M. 1995 ApJ 448, 788

Nota, A., Pasquali, A., Marston, A.P., Lamers, H.J.G.L.M., Clampin, M. \& SchulteLadbeck, R.E. 2002 AJ 124, 2920

Oudmaijer, R. D., Proga, D., Drew, J. E. \& de Winter, D. 1998 MNRAS 300, 1700

Parthasarathy, M., Jain, S.K. \& Bhatt, H.C. 2000 A\&A 355, 221

Schulte-Ladbeck, R.E. 1997 Rev. Modern Astron. 10, 135

Schulte-Ladbeck, R.E., Clayton, G.C., Hillier, J.D., Harries, T.J. \& Howarth, I.D. 1994 ApJ 429, 846

Schulte-Ladbeck, R.E, Leitherer, C., Clayton, G.C., Robert, C., Meade, M.R., Drissen, L., Nota, A. \& Schmutz, W. 1993 ApJ 407, 723

Skinner, S.L., Zhekov, S.A., Güdel, M. \& Schmutz, W. 2002 579, 764

Smith, N. \& Davidson, K. 2001 ApJ 551, L101

Vreux, J.-M., Gosset, E., Bohannan, B. \& Conti, P.S. 1992 A\&A 256, 148

Woosley, S.E. \& Heger, A. 2004, these proceedings

Zickgraf 1985 Ph.D. Thesis, University of Heidelberg

Zickgraf, F.-J. 1998, Non-spherical stellar winds in the post-main sequence evolution of massive stars, Habilitationsschrift (Heidelberg)

http://www.hs.uni-hamburg.de//DE/Ins/Per/Zickgraf/habil.html

Zickgraf, F.-J. 2001 A\&A 375, 122

Zickgraf, F.-J. \& Schulte-Ladbeck, R.E. 1989 A\&A 214, 274

Zickgraf, F.-J., Wolf, B., Stahl, O., Leitherer, C., Appenzeller, I. 1986 A\&A 163, 119 